\title{
A Study to Assess the Association between Umbilical Cord Coiling Index and Perinatal Outcomes
}

\author{
Purnima Satoskar ${ }^{1}$, Anamika Singh ${ }^{2}$, Hemraj Narkhede ${ }^{3}$
}

\begin{abstract}
Aim: To analyze whether antenatal second trimester umbilical coiling index measurement could predict adverse perinatal outcome. Materials and methods: A study designed was a prospective observational study, enrolled total 100 singleton pregnancies, who were fulfilling inclusion criteria, attending regular antenatal checkup and willing for institutional deliveries. Each case was evaluated by sonographic study for umbilical coiling index at the time of routine fetal anatomical survey between 18 weeks and 24 weeks and then followed up for evaluation of perinatal outcome.

Results: In present study, $79 \%$ had normocoiling while $10 \%$ had hypocoiling and $11 \%$ had hypercoiling of cord. Of these, $5 \%$ had preterm deliveries which was statistically significant $(p=0.046)$. Also, meconium-stained liquor was noted in $10 \%$ cases, among which $50 \%$ had abnormal coiling index. This was statistically significant $(p=0.008)$.

Conclusion: Measuring coiling index can help in predicting adverse perinatal outcomes resulting in closer fetal monitoring and improved fetal outcome.

Clinical significance: Antenatal umbilical cord coiling index (aUCI) is an important parameter to be studied during second trimester which may help to screen patients at risk of adverse perinatal outcome.

Keywords: Measuring coiling index, Perinatal outcome, Umbilical cord.

Journal of South Asian Federation of Obstetrics and Gynaecology (2019): 10.5005/jp-journals-10006-1697
\end{abstract}

\section{INTRODUCTION}

Umbilical cord, derived from Latin word funiculus umbilicalis, is a narrow cord of tissue that connects a developing embryo or fetus with the placenta.' Umbilical cord has three blood vessels and it is susceptible to kinking, torsion and compression which may affect the perinatal outcome. Normally, these vessels are encased by Wharton's jelly. Also, amniotic fluid and helical coiling of blood vessels provide protection to them. This helical arrangement of umbilical vessels is called spiral course. ${ }^{2}$

A coil is defined as having completed a $360^{\circ}$ spiral course of umbilical vessel around Wharton's jelly. This coiling property of umbilical cord was first described by Berengarius in $1521 .^{3}$ In 1954, umbilical coiling was first measured by Edmonds who divided the total number of coils by umbilical cord length in centimeters and called it "Index of twist." He allocated positive and negative scores to clockwise and anticlockwise coiling, respectively. ${ }^{3,4}$ Later, Strong et al. simplified it by removing three directional score and named it "The umbilical cord coiling index," (UCI). ${ }^{3,5}$ An abnormal $\mathrm{UCl}$ can be both hypocoiled cords ( $\mathrm{UCl}<10$ th percentile) or hypercoiled cords ( $\mathrm{UCl}>90$ th percentile). These abnormal coiling index has been reported to equate with adverse perinatal outcome. ${ }^{2-4,6}$

Normal value of coiling index is calculated approximately as 1 coil $/ 5 \mathrm{~cm}$ of umbilical cord length or $0.20-0.24$ coils $/ \mathrm{cm} .{ }^{5}$ Antenatal umbilical cord coiling index is calculated as the reciprocal of the distance between a pair of coils measured in centimeters. The distance can be measured from the inner edge of an arterial or venous wall to the outer edge of next coil along the same side of umbilical cord. ${ }^{6}$ The final value is taken as the average of three readings at different segments along the entire length of umbilical cord. It is assumed that the umbilical coiling is entirely developed by the end of first trimester and does not alter thereafter. On the other hand the cord lengthens between the already established
${ }^{1}$ Department of Obstetrics and Gynecology, Seth Gordhandas Sunderdas Medical College and Nowrosjee Wadia Maternity Hospital, Mumbai, Maharashtra, India

${ }^{2}$ Department of Obstetrics and Gynecology, Government Medical College and Hospital, Nagpur, Maharashtra, India

${ }^{3}$ Department of Obstetrics and Gynecology, Postgraduate Institute, Yashwantrao Chavan Memorial Hospital, Pune, Maharashtra, India

Corresponding Author: Anamika Singh, Department of Obstetrics and Gynecology, Government Medical College and Hospital, Nagpur, Maharashtra, India, Phone: +91 9082208509, e-mail: dr.anamika27. AS@gmail.com

How to cite this article: Satoskar P, Singh A, Narkhede H. A Study to Assess the Association between Umbilical Cord Coiling Index and Perinatal Outcomes. J South Asian Feder Obst Gynae 2019;11(4):243-245.

Source of support: Nil

Conflict of interest: None

coils, ${ }^{4}$ thus it can be said that the true $\mathrm{UCl}$ can be predicted with accuracy from the ultrasonographic assessment in the second trimester. ${ }^{7}$ In the present study at 18-24 weeks period of gestation, aUCl was determined at routine fetal anatomy survey scans and its association with perinatal outcomes studied.

\section{Materials and Methods}

After receiving approval from Institutional Ethical Committee and patients consent, this prospective observational study was carried out in the Department of Obstetrics and Gynecology at a Tertiary Care Hospital in Mumbai over a period of 18 months. Total 100 patients with singleton pregnancy between 18 weeks and 24 weeks and maternal age between 18 years and 35 years were included in the study. 
Patients with multifetal gestation, malformed fetus, single umbilical artery, high risk pregnancies complicated with maternal diseases like hypertension, infections, diabetes, thrombophilia and patients with personal history like smoking and drug abuse and those unwilling to participate in the study were excluded from the study.

Patients satisfying inclusion criteria, visiting for regular antenatal checkups and willing for institutional deliveries were evaluated by sonographic study for umbilical coiling index at the time of routine fetal anomaly scan between 18 weeks and 24 weeks. A single sonologist performed all the anomaly scans to rule out interobserver variation of coiling index.

In a second trimester ultrasonography (USG), umbilical cord was evaluated and longitudinal cord images were recorded for calculation of cord coiling. The distance between the coils was measured using built-in calipers from the inner edge of an arterial or venous wall to the outer edge of the next coil along one side of the umbilical cord. Three readings were taken and average of three was calculated. The aUCI was calculated as a reciprocal value of the distance between a pair of coils (aUCI $=1$ /distance in centimeters).

Perinatal outcomes were assessed in terms of fetal respiratory distress, meconium-stained liquor, low birth weight delivery, preterm delivery (gestational age $<37$ weeks), Apgar score (Apgar score less than 7 was taken as low Apgar score) and neonatal intensive care unit (NICU) admissions (baby requiring resuscitation by AMBU bag and NICU care for observation or ventilator support was taken in NICU admission).

\section{Statistical Analysis}

Data analysis was conducted using professional statistics package EPI Info 7.0 version for windows. Appropriate tests of significance were applied depending on the character and distribution of variables like Chi-square test, Fisher's exact test for categorical variables. Values of $p<0.05$ was considered statistically significant.

\section{Results}

Out of 100 cases, 79 of the subjects showed normal coiling index, 10 with hypocoiling and 11 with hypercoiling of the umbilical cord. A total of 59 cases were primigravida with 13 showing abnormal coiling index while among 41 multigravidas only 8 had abnormal coiling (Table 1). This data was rather insignificant making clear that coiling index might not alter with parity ( $p$ value $=0.546$ ). Among 21 patients with abnormal coiling pattern, 5 patients had preterm deliveries (14.2\%) and of 79 patients with normal coiling, 2 had preterm delivery (2.5\%) ( $p$ value $=0.046$ ). Out of 10 patients who delivered with meconium-stained liquor 4 showed hypocoiling and 1 had hypercoiling (Table 1). This data was statistically significant proving that hypocoiling can be strongly associated with meconium-stained liquor ( $p$ value $=0.001$ ). A total of 75 patients delivered vaginally; 25 patients delivered by lower segment cesarean section. Most common indication for lower segment cesarean section was meconium-stained liquor and fetal distress. Present study shows that abnormal coiling of cord could not predict the mode of delivery. Different cord insertions observed were: central in 75 placentas, eccentric in 14 and marginal in 11 placentas as shown in Table 1.

Among total cases, 19 patients had intrauterine growth restriction (IUGR). Out of these four had hypocoiling and five had hypercoiling of cord. A total of 81 patients delivered appropriate for gestational age (AGA) baby out of which 7 showed hypocoiling and 5 showed hypercoiling (Table 1). The present study could not prove any association between abnormal coiling and IUGR ( $p$ value $=0.57$ ). Further studies with larger sample size are needed to study this association.

Only one newborn died during early neonatal period. Perinatal outcome was evaluated in the form of Apgar score and adverse events developed in neonates. Apgar score of 7-10 at the 1st minute is considered to be normal. Table 2 shows the frequency of Apgar score at 1 and 5 minutes. There was no difference among the three groups in Apgar score at 1 and 5 minutes as shown in Table 3.

\section{Discussion}

It is now justified that there is clinical correlation between perinatal outcome and umbilical cord coiling. Several trials had been conducted in the past which have shown the relationship between perinatal outcome and the umbilical cord coiling. Similarly, in the present study, perinatal factors like meconium staining, birth weight, Apgar score at 1 minute, mode of delivery, NICU admission, gestational age, fetal respiratory distress was correlated with umbilical cord coiling.

The women included in present study were ranging from 18 years to 35 years of age. Majority of them were in age group 25-29

Table 1: Comparison of perinatal factors among different umbilical cord coiling index groups

\begin{tabular}{|c|c|c|c|c|c|c|}
\hline \multirow[b]{2}{*}{ Perinatal factors } & & \multicolumn{3}{|c|}{ UClgroup } & \multirow[b]{2}{*}{ Total } & \multirow[b]{2}{*}{$p$ value } \\
\hline & & $\begin{array}{l}<10 \text { th percentile } \\
\text { (hypocoiled) }\end{array}$ & $\begin{array}{l}\text { 10th-90th percentile } \\
\text { (normal coiled) }\end{array}$ & $\begin{array}{l}\text { >90th percentile } \\
\text { (hypercoiled) }\end{array}$ & & \\
\hline \multirow[t]{2}{*}{ Gravida } & Primigravida & 8 & 46 & 5 & 59 & 0.546 \\
\hline & Multigravida & 3 & 33 & 5 & 41 & \\
\hline \multirow[t]{2}{*}{ Maturity at birth } & Full term & 10 & 77 & 8 & 95 & 0.046 \\
\hline & Preterm & 1 & 2 & 2 & 5 & \\
\hline \multirow[t]{2}{*}{ Liquor color } & Clear & 7 & 74 & 9 & 90 & 0.008 \\
\hline & MSAF & 4 & 5 & 1 & 10 & \\
\hline \multirow[t]{2}{*}{ Delivery } & Normal & 6 & 61 & 8 & 75 & 0.247 \\
\hline & LSCS & 5 & 18 & 2 & 25 & \\
\hline \multirow[t]{2}{*}{ Birth weight } & $<10$ th centile & 4 & 42 & 5 & 51 & 0.57 \\
\hline & $>10$ th centile & 7 & 37 & 5 & 49 & \\
\hline \multirow[t]{3}{*}{ Cord attachment } & Central & 15 & 58 & 2 & 75 & - \\
\hline & Eccentric & 2 & 11 & 1 & 14 & \\
\hline & Marginal & 1 & 9 & 1 & 11 & \\
\hline
\end{tabular}


Table 2: Apgar score at 1 minute and 5 minutes

\begin{tabular}{lccc}
\hline Apgar score & Apgar value & Frequency & Percent \\
\hline Apgar 1 minute & $<7$ & 4 & 4.0 \\
& 7 & 8 & 8.0 \\
& 8 & 88 & 88.0 \\
Apgar 5 minutes & $<7$ & 1 & 1.0 \\
& 7 & 1 & 1.0 \\
& 8 & 5 & 5.0 \\
& 9 & 93 & 93.0 \\
\hline
\end{tabular}

Table 3: Comparison of Apgar score (at 1 and 5 minutes) among different umbilical cord coiling index groups

\begin{tabular}{lllll}
\hline Apgar score & UCl groups & $N$ & Mean & pvalue \\
\hline Apgar-1 & <10th percentile & 11 & $7.91 \pm 0.302$ & 0.955 \\
& 10th-90th percentile & 79 & $7.94 \pm 0.462$ & \\
& >90th percentile & 10 & $7.90 \pm 0.316$ & \\
Apgar-5 & $<$ 10th percentile & 11 & $9.00 \pm 0.000$ & 0.878 \\
& 10th-90th percentile & 79 & $8.95 \pm 0.450$ & \\
& >90th percentile & 10 & $9.00 \pm 0.000$ & \\
\hline
\end{tabular}

years. This had no statistical significance. Distribution of gravidity shows that the majority of women were primigravida of these $46 \%$ had normocoiling, $8 \%$ had hypocoiling and $5 \%$ had hypercoiling ( $p$ value-0.546). 25\% underwent LSCS out of these $72 \%$ had normocoiling, $20 \%$ had hypocoiling, $8 \%$ had hypercoiling. $75 \%$ underwent vaginal delivery and $81.3 \%$ had normocoiling, $8 \%$ had hypocoiling and $10.6 \%$ had hypercoiling ( $p$ value -0.247 ), thus the mode of delivery could not be predicted by coiling index.

A meta-analysis conducted by Gupta et al. showed that hypocoiling is associated with increased incidence of fetal demise, intra partum fetal heart rate decelerations, operative delivery, fetal distress and chorioamnionitis. ${ }^{3}$ In current study, birth weight was correlated to umbilical cord coiling. It was found that out of 100 babies delivered, 51 were found to be IUGR, i.e., $<2.5 \mathrm{~kg}$, among which $82.4 \%$ had norm coiling, $7.8 \%$ had hypocoiling and $9.8 \%$ had hypercoiling. Rest 49 babies with normal birth weight showed normocoiling in $75.5 \%, 14.2 \%$ showed hypocoiling and $10.3 \%$ showed hyper coiling. The $p$ value $(0.57)$ for this was statistically insignificant, thus abnormal coiling index cannot help in predicting higher incidences of IUGR. But we found significant association between abnormal coiling and preterm labor ( $p$ value $=0.046$ ). Thus, measuring coiling index might be helpful in having suspicion for preterm labor and with close fetal monitoring adverse perinatal outcome could be predicted. This result of present study was compared with the study done by Rana et al. ${ }^{2}$ and Raio et al. ${ }^{8}$

Out of the total cases, $10 \%$ had meconium-stained liquor of which $50 \%$ were with normal coiling, $40 \%$ with hypocoiling, and $10 \%$ with hypercoiling. Total $90 \%$ had clear liquor of which $82.2 \%$ showed normal coiling, $7.8 \%$ showed hypocoiling, and $10 \%$ showed hypercoiling. Thus, helping in proving that abnormal coiling can be associated with MSAF ( $p$ value $=0.042$ ). Similar results were noted in previous studies. ${ }^{3,5,9-11}$

Apgar at 1 minute and 5 minutes was not found to be statistically significant. But other similar umbilical cords studies ${ }^{3,9,12}$ showed that under coiling was associated with low Apgar scores. Padmanabhan et al. ${ }^{9}$ studied 130 umbilical cords and found that hypocoiling of cord was associated with low Apgar scores. Indeed, over-coiling per se, even without cord stricture, alone has been documented at autopsy in $23 \%$ of unexplained fetal deaths. ${ }^{13}$

\section{Conclusion}

From the results of present study, we concluded that abnormal coiling index can be associated with preterm labor and by closely monitoring these high-risk fetuses, such adverse outcomes can be declined. Also, study found that hypocoiling might lead to meconium-stained amniotic fluid. Hence measuring coiling index can help in predicting adverse perinatal outcomes resulting in closer fetal monitoring and improved fetal outcome.

\section{Clinical Significance}

Antenatal umbilical cord coiling index can prove to be a very helpful aid during the second trimester to screen patients at risk of adverse perinatal outcome.

\section{References}

1. Dyawapur A. A correlational study to assess the umbilical cord coiling index and perinatal outcomes in selected hospitals at Bijapur. IOSR J Nurs Health Sci 2016;5(4):82-87. DOI: 10.9790/1959-0504038287.

2. Rana J, Ebert GA, Kappy KA. Adverse perinatal outcome in patients with an abnormal umbilical coiling index. Obstet Gynecol 1995;85(4):573-577. DOI: 10.1016/0029-7844(94)00435-G.

3. Gupta S, Faridi MMA, Krishnan J. Umbilical coiling index. J Obstet Gynecol India 2006;56(4):315-319.

4. Edmonds HW. The spiral twist of normal umbilical cords in twins and singletones. Am J Obstet Gynecol 1954;67(1):102-120. DOI: 10.1016/0002-9378(54)90201-X.

5. Strong $T H$, Jarles DL, Vega JS, et al. The umbilical coiling index. Am J Obstet Gynecol 1994;170(1 Pt 1):29-32. DOI: 10.1016/S00029378(94)70378-7.

6. Lacro RV, Jones KL, Benirschke K. The umbilical cord twist: origin, direction, and relevance. Am J Obstet Gynecol 1987;157(4 Pt 1): 833-838. DOI: 10.1016/S0002-9378(87)80067-4.

7. Qin Y, Lau TK, Rogers MS. Second trimester ultrasonographic assessment of the umbilical coiling index. Ultrasound Obstet Gynecol 2002;20(5):458-463. DOI: 10.1046/j.1469-0705.2002.00846.x.

8. Raio L, Ghezzi F, Di Naro E, et al. Prenatal diagnosis of a lean umbilical cord: a simple marker of the fetuses at risk. Ultrasound Obstet Gynecol 1999;13(3):157-160. DOI: 10.1046/j.1469-0705.1999.13030157.x.

9. Padmanabhan LD, Mhaskar R, Mhaskar A. Umbilical vascular coiling and the perinatal outcome. J Obstet Gynecol India 2001;51(6):43-44.

10. Devaru D, Meghna T. Umbilical coiling index \& the perinatal outcome. J Obstet Gynecol India 2012;62(1):43-46. DOI: 10.1007/s13224-0120158-4.

11. Nakamura M, Hasegawa J, Matsuaka R. Risk analysis for the birth of a small for gestational age (SGA) infant. Internet Journal of Gynecology and Obstetrics 2010;13(2):1-5.

12. de Laat MW, Franx A, Bots ML, et al. Umbilical coiling index in normal and complicated pregnancies. Obstet Gynecol 2006;107(5): 1049-1055. DOI: 10.1097/01.AOG.0000209197.84185.15.

13. Peng $H Q$, Levitin-Smith $M$, Rochelson $B$, et al. Umbilical cord stricture and over-coiling are common causes of fetal demise. Pediatr Dev Pathol 2006;9(1):14-19. DOI: 10.2350/05-05-0051.1. 